\title{
Clinical outcomes in patients with stage I non-seminomatous germ cell cancer
}

\author{
Zhao-Jie Lv ${ }^{1,2 *}$, Song $\mathrm{Wu}^{1,3,4 *}$, Pei Dong ${ }^{3}$, Kai Yao ${ }^{3}$, Yin-Yin He ${ }^{5}$, Yao-Ting Gui ${ }^{5}$, Fang-Jian Zhou ${ }^{3}$, Zhuo-Wei Liu ${ }^{3}$ \\ and Zhi-Ming $\mathrm{Cai}^{1,5}$
}

This study assesses the long-term outcomes in Han Chinese patients with clinical stage I non-seminomatous germ cell testicular cancer (CSI NSGCT) treated with surveillance, retroperitoneal lymph node dissection (RPLND) and adjuvant chemotherapy. We retrospectively evaluated 89 patients with a mean age of 26.5 years. After orchiectomy, 37 patients were treated with surveillance, 34 underwent RPLND and 18 were managed with chemotherapy. The overall survival rate, the recurrence-free survival rate and the risk factors were evaluated. The median follow-up length was 92 months (range: 6-149 months). Thirteen of the 89 patients (14.6\%) had relapses, and one died by the evaluation date. The overall survival rate was $98.9 \%$. The cumulative 4-year recurrence-free rates were $80.2 \%, 92.0 \%$ and $100 \%$ for the surveillance, RPLND and chemotherapy groups, respectively. The disease-free period tended to be briefer in patients with a history of cryptorchidism and those with stage Is. Therefore, surveillance, RPLND and adjuvant chemotherapy might be reliable strategies in compliant patients with CSI NSGCT. Surveillance should be recommended for patients with the lowest recurrence rate, especially those without lymphovascular invasion. This study might aid the establishment of a standard therapy for CSI NSGCT in China.

Asian Journal of Andrology (2013) 15, 558-563; doi:10.1038/aja.2013.16; published online 20 May 2013

Keywords: chemotherapy; clinical stage I non-seminomatous germ cell testicular cancer (CSI NSGCT); outcome; retroperitoneal lymph node dissection (RPLND); surveillance; treatment protocols

\section{INTRODUCTION}

The worldwide incidence of testicular cancer has doubled over the last 40 years. This cancer constitutes $2 \%$ of all malignancies. ${ }^{1}$ In 2012,8590 new cases of testicular cancer were estimated to be diagnosed in the United States. ${ }^{2}$ The incidence rate ranges from approximately $1 / 100000$ in China to 9.2/100 000 in Denmark. ${ }^{3}$ The majority of testicular cancer cases occur between the ages of 15 and 34 years. ${ }^{4}$ Testicular cancer is classified into two major types: seminomas and non-seminomatous germ cell tumours (NSGCTs). NSGCTs include yolk sac tumour, embryonal carcinoma, choriocarcinoma and teratoma with mature or immature elements. Approximately $60 \%$ of patients with NSGCTs are in clinical stage I at diagnosis. ${ }^{5}$ Treatments include surveillance, retroperitoneal lymph node dissection (RPLND) and adjuvant chemotherapy after initial orchiectomy. All management protocols yield cancer-specific survival rates of $98 \%-99 \%$ with or without risk factors. ${ }^{6,7}$

Currently, selecting the appropriate treatment for clinical stage I non-seminomatous germ cell testicular cancer (CSI NSGCT) remains controversial. According to the National Comprehensive Cancer Network, surveillance was recommended for patients with a low recurrence risk. The relapse rate for this strategy is approximately $30 \%$. For patients who cannot manage the psychological distress of recurrence, chemotherapy or RPLND is possible option. No consensus exist regarding which strategy should be preferred in China. ${ }^{8-11}$

The current challenge in managing CSI NSGCT is to achieve a cure with minimal acute or long-term toxicity related to treatment. The inherent risks of treatment are associated with radiation exposure through repeated computed tomography scans, the added morbidity of chemotherapy-induced cardiopulmonary toxicities and secondary malignancies. ${ }^{12-14}$ Patients treated for testicular cancer have a higher risk of ejaculatory, orgasmic and erectile dysfunction (ED) compared with healthy males. A meta-analysis revealed that ED was reported in $11.5 \%$ of the available retrospective studies and in $14 \%$ of the prospective studies. ${ }^{15}$ Several studies have reported on the prognostic factors concerning occult metastatic disease in patients with CSI NSGCT, but the long-term oncological results with regard to Han Chinese patients remain unknown.

This study retrospectively reviews the patients with CSI NSGCT treated at the urological department of Shenzhen Second People's Hospital, Shenzhen PKU-HKUST Medical Centre and Sun Yat-Sen University Cancer Centre in Guangdong, China, from 1997 to 2011. This study evaluates the long-term outcomes of the applied treatment strategies of surveillance, RPLND and adjuvant chemotherapy in Han Chinese patients.

\footnotetext{
${ }^{1}$ Shenzhen Second People's Hospital, the First Affiliated Hospital of Shenzhen University, Shenzhen 518036, China; ${ }^{2}$ Anhui Medical University, Hefei 230032, China; ${ }^{3}$ Department of Urology, Sun Yat-Sen University Cancer Centre, Guangzhou 510060, China; ${ }^{4}$ Institute of Immunology, Zhongshan School of Medicine, Sun Yat-Sen University, Guangzhou 510060, China and ${ }^{5}$ Institute of Urology, Shenzhen PKU-HKUST Medical Centre, Shenzhen 518036, China

* These authors contributed equally to the work.

Correspondence: Dr ZM Cai (caizhiming2000@yahoo.com) and Dr ZW Liu (liuzhw@sysucc.org.cn)

Received: 21 November 2012; Revised: 3 January 2013; Accepted: 26 January 2013; Published online: 20 May 2013
} 
Table 1 Clinical characteristics of patients with clinical stage I nonseminomatous germ cell tumour

\begin{tabular}{|c|c|c|}
\hline Variable & No. & $\%$ \\
\hline \multicolumn{3}{|l|}{ Age (year) } \\
\hline Mean \pm s.d. & $26.2 \pm 13.4$ & \\
\hline Range & $0.3-66$ & \\
\hline \multicolumn{3}{|l|}{ Local side } \\
\hline Right & 49 & 55.0 \\
\hline Left & 39 & 43.8 \\
\hline Both sides & 1 & 1.1 \\
\hline \multicolumn{3}{|l|}{ History of cryptorchidism } \\
\hline Yes & 6 & 6.7 \\
\hline No & 83 & 93.2 \\
\hline \multicolumn{3}{|l|}{ Primary tumour stage } \\
\hline la & 40 & 44.9 \\
\hline $\mathrm{Ib}$ & 12 & 13.5 \\
\hline Is & 37 & 41.6 \\
\hline \multicolumn{3}{|l|}{ Pathophysiology } \\
\hline Embryonal carcinoma & 14 & 15.7 \\
\hline Teratoma & 18 & 20.2 \\
\hline Choriocarcinoma & 1 & 1.10 \\
\hline Yolk sac tumour & 16 & 18.0 \\
\hline Mixed tumours & 40 & 44.9 \\
\hline \multicolumn{3}{|l|}{ Initial treatment } \\
\hline Chemotherapy & 18 & 20.2 \\
\hline Surveillance & 37 & 41.6 \\
\hline RPLND & 34 & 38.2 \\
\hline \multicolumn{3}{|c|}{ IIEF-5 score ( $\leqslant 7, n=59$, time $=2$ months $)$} \\
\hline Chemotherapy & 3 & 21.4 \\
\hline Surveillance & 5 & 21.7 \\
\hline RPLND & 4 & 18.2 \\
\hline
\end{tabular}

Abbreviations: IIEF-5: International Index of Erectile Function (five-item); RPLND, retroperitoneal lymph node dissection.

\section{MATERIALS AND METHODS}

\section{Patients}

Of the 492 consecutive testicular cancer patients treated at Shenzhen Second People's Hospital, Shenzhen PKU-HKUST Medical Centre and Sun Yat-Sen University Cancer Centre, 205 were diagnosed with non-seminomatous tumours between January 1997 and December 2011. All the patients were Han Chinese and 89 patients (43.4\%) were in stage I. Forty of the 89 patients $(44.9 \%)$ were in stage Ia. Twelve $(13.5 \%)$ were in stage Ib, and $37(41.6 \%)$ were in stage Is. The median age of the patients was 26.5 years (range: 1-66 years) at the time of orchiectomy. Thirty-nine were primary right tumours and 49 were on the left side. One patient had tumours in both testicles (Table 1).

The patients underwent initial clinical staging at the time of orchiectomy. A history, a physical examination and a computed tomography scan of the thorax, abdomen and pelvis (at 5-mm intervals) confirmed the clinical staging. An assay of serum tumour markers including $\beta$-human chorionic gonadotropin, $\alpha$-fetoprotein and lactic dehydrogenase were assessed before and after orchiectomy and weekly throughout the follow-up period until the final clinical staging. Stage Is was defined as persistent elevated serum tumour markers without clinical or radiographic evidence of disease. A restaging 7-8 weeks after orchiectomy confirmed this diagnosis. Definitive staging was defined according to the American Joint Committee on Cancer stage groupings (Table 2).

\section{Treatments}

After the orchiectomy and staging, each patient was given a thorough explanation of the advantages and disadvantages of the three treatment alternatives (surveillance, RPLND and adjuvant chemotherapy). The treatment options were recommended based on the National Comprehensive Cancer Network guidelines (2012). ${ }^{4}$ A vascular or lymphatic invasion of the primary tumour was used to discriminate between the low-risk and high-risk patients. The patients were offered the choice of treatment.

If vascular or lymphatic invasion was not present or there was less than $50 \%$ embryonal carcinoma, then surveillance was recommended with strict follow-ups.

RPLND was suggested for the patients with predominant teratomas or for those who were opposed to chemotherapy or surveillance. Primary modified retroperitoneal lymph node dissection was performed for the patients who entered the RPLND programme. The surgery for right-sided tumours included the paracaval, precaval, upper interaortocaval, para-aortic and right iliac node. For left-sided tumours, the para-aortic, upper interaortocaval, upper pre-aortic and left iliac areas were resected. ${ }^{16,17}$ Dissection below the origin of the inferior mesenteric artery was not performed unless a palpable mass was present in this area. If the frozen sections of the lymph node showed presence of a tumour, a bilateral RPLND was conducted. The ipsilateral spermatic vein was removed in all cases. If the RPLND results were negative, then no adjuvant chemotherapy was recommended. If the RPLND results were positive, then adjuvant chemotherapy was advised.

Adjuvant chemotherapy was the treatment of choice for the patients with vascular invasion, lymphatic invasion, more than $50 \%$ embryonal carcinoma or some combination thereof. Adjuvant chemotherapy treatment included two cycles of the bleomycin, etoposide, cisplatin (BEP) regimen, which consisted of $30 \mathrm{mg}$ of bleomycin on days 1, 5 and 15 , over a cycle length of 21 days, $100 \mathrm{mg} \mathrm{m}^{-2}$ of etoposide on days 1 through 5 and $25 \mathrm{mg} \mathrm{m}^{-2}$ of cisplatin on days 1 through 4 . Shortterm adjuvant chemotherapy was recommended for the high-risk patients and they were able to choose between one or two courses of $\mathrm{BEP}$, whereas the low-risk patients were able to choose either surveillance or one course of BEP. Chemotherapy with BEP for three cycles was required for the stage Is patients (Figure 1).

Table 2 Definition table of the clinical stages: American Joint Committee on Cancer (AJCC) Stage Groupings

\begin{tabular}{|c|c|c|}
\hline Clinical stages & Definition & TNM stage \\
\hline Stage 0 (S 0) & Carcinoma in situ & pTis \\
\hline Stage I (S I) & Cancer has not spread to either lymph nodes or other organs & pT1-4, NO, MO, SX \\
\hline Stage la (S la) & Cancer has spread to retroperitoneal lymph nodes, with normal or slightly high level of serum tumour markers & pT1, N0, MO, SO-1 \\
\hline Stage Ib (S Ib) & $\begin{array}{l}\text { Cancer has invaded the outer membrane surrounding the testicle, blood or lymphatic vessels within the testicle, } \\
\text { or has spread to the spermatic cord or the scrotum, with normal serum markers }\end{array}$ & pT2-4, NO, MO, SO \\
\hline Stage Is (S Is) & Serum tumour markers are persistent elevated without clinical or radiographic evidence of disease & pT1-4, N0, MO, S1-3 \\
\hline Stage II (S II) & Cancer has spread to any number of regional lymph nodes but not to lymph nodes in other parts of the body or distant organs & pT1-4, N1-3, M0, SX \\
\hline Stage III (S III) & Cancer has spread to distant lymph nodes or to any organ & pT1-4, N0-3, M1, SX \\
\hline
\end{tabular}




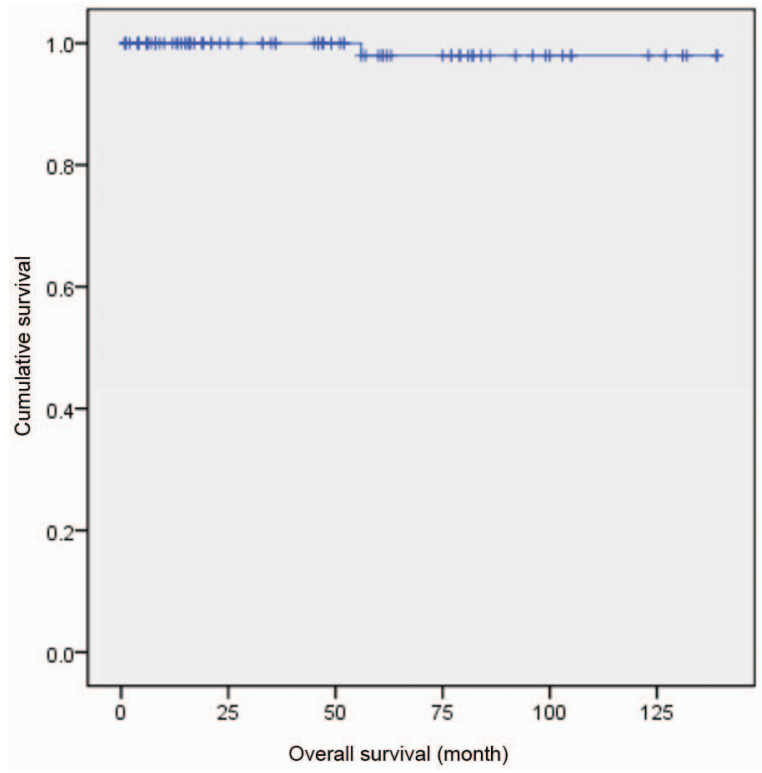

Figure 1 Treatment algorithm after orchiectomy according to individual risk factors in patients with clinical stage I non-seminomatous germ cell tumours. High risk: vascular invasion or lymphatic invasion; low risk: none vascular invasion and none lymphatic invasion. DOD: died of disease; ER: evidence of recurrence; NER: no evidence of recurrence; RPLND: retroperitoneal lymph node dissection.

\section{Follow-up visits}

The patients were observed using a standardized follow-up protocol. They were seen at bimonthly intervals in year 1 , every 3 months in year 2, every 4 months in year 3 and every 6 months thereafter. The minimum follow-up length was 5 years, but 10 -year periods were recommended. Physical examinations, serum tumour markers and chest X-rays were obtained at each outpatient visit. Abdominal and pelvic computed tomography scans were performed at every other visit. Routine abdominal and pelvic imaging

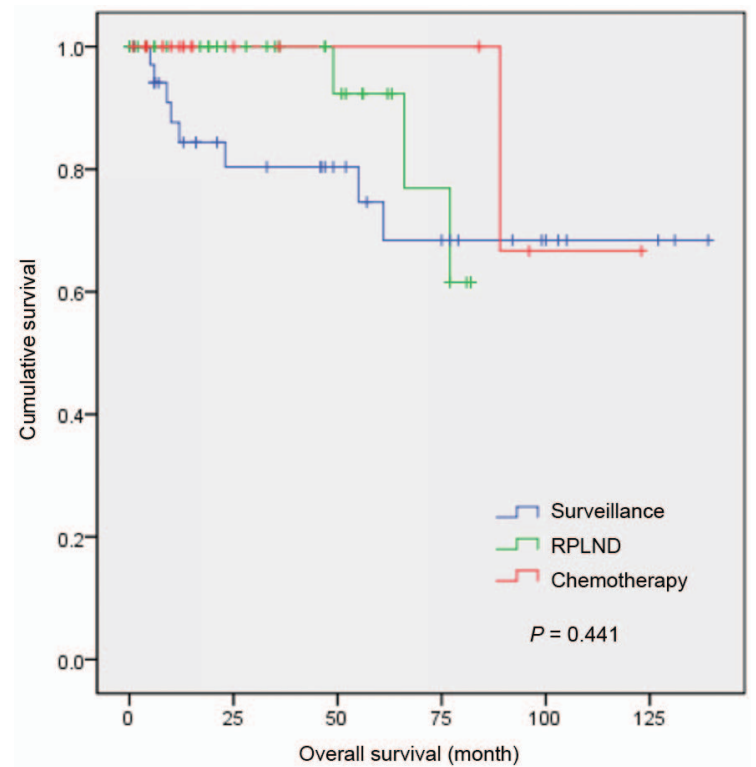

Figure 2 The overall survival curve of CSI NSGCT (89 patients). CSI NSGCT, clinical stage I non-seminomatous germ cell testicular cancer.

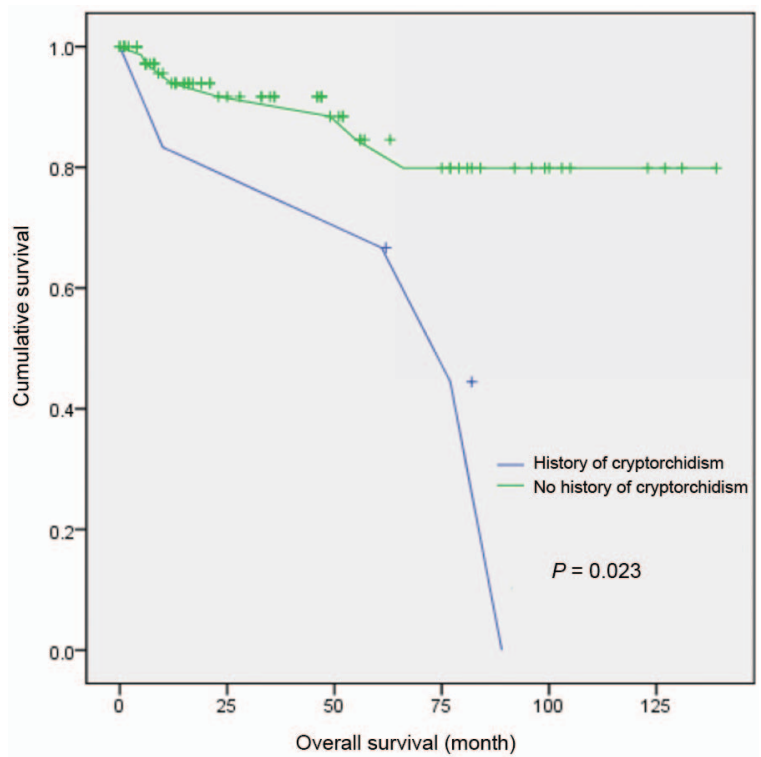

Figure 3 Recurrence-free survival for patients with CSI NSGCT treated with surveillance, RPLND and adjuvant chemotherapy. CSI NSGCT, clinical stage I non-seminomatous germ cell testicular cancer; RPLND, retroperitoneal lymph node dissection.

was not mandatory after 5 years unless the primary tumour showed elements of mature teratoma. Surgery was recommended for the patients with a retroperitoneal relapse larger than $2 \mathrm{~cm}$, those who were suspected of growing a teratoma, or both. No other therapy was provided when the tumour was completely removed. Those who relapsed or developed metastases that were detected early received at least three courses of chemotherapy.

The International Index of Erectile Function 5-item questionnaire was routinely used to evaluate the patients for sexual function 2 months after surgery. Disease progression was defined using imaging physical examination evidence, elevated serum tumour markers or

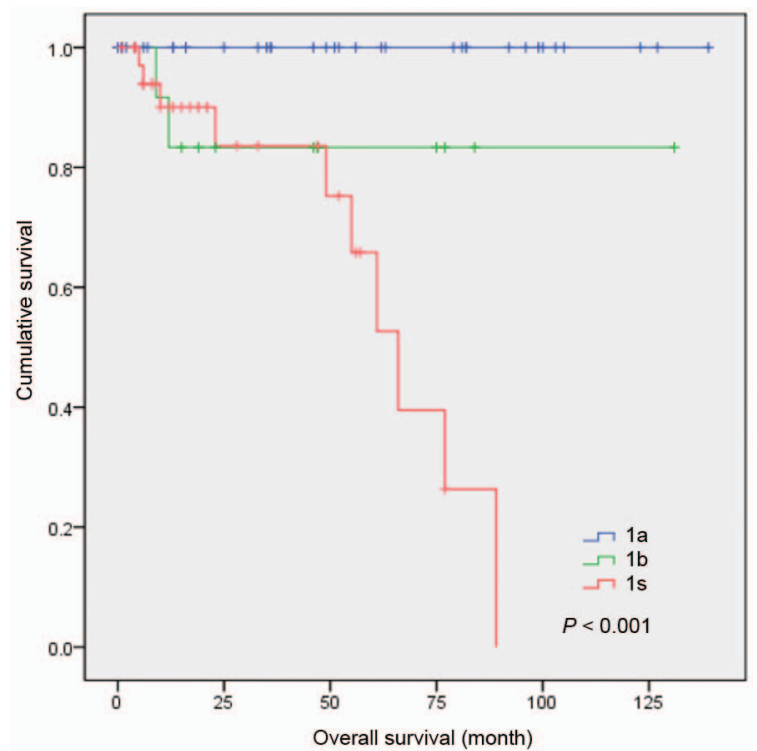

Figure 4 Recurrence-free survival for six patients with history of cyptorchidism and 83 patients without history of cryptorchism. 
both. Acute chemotherapy-related toxicity including haematological, renal, gastrointestinal, pulmonary, neurological toxicity and infectious complications was also evaluated at the follow-up visit. ${ }^{11}$

\section{Statistical analysis}

The patient data were evaluated across a documented follow-up period until February 2011. One man was lost to follow-up. The evaluation included the overall survival rate, the recurrence rate, the relapse time and the predictive risk factors for recurrence. Summary statistics were used to describe the patient sample. Continuous variables were compared using paired $t$-tests, and categorical variables were compared using Fisher's exact test. The Kaplan-Meier method was used to estimate the overall and recurrence-free survival rate calculated from orchiectomy date until death or from the last known date the patient was alive. Survival curves were compared using the log-rank test. Cox's proportional hazard multivariate regression model was used to explore the effect of the clinicopathological variables on the survival rate. Factors in the multivariate analysis included the patient's age at surgery, the tumour side, the initial treatment after orchiectomy, the history of cryptorchidism and the clinical primary tumour stage. The SPSS (Statistical Package for the Social Sciences) 17.0 (SPSS Inc., Chicago, IL, USA) was used for all data analyses, and $P<0.05$ was considered significant.

\section{RESULTS}

Eighty-nine patients with CSI NSGCT who were treated at Sun Yat-sen University Cancer Center between 1997 and 2011 were identified. The median follow-up visit was 92 months after surgery (range: 6-149 months). The 5-year overall survival rate was $98.9 \%$. At the evaluation date, 13 of the 89 patients (14.6\%) had suffered relapses, and one patient had died. This patient, who was initially on RPLND, showed a histological workup of pT3/Is and died of liver cancer 56 months after treatment (Figure 2).

Figure 3 shows the recurrence-free survival rate of the surveillance, RPLND and adjuvant chemotherapy groups. In surveillance group, eight of the 37 patients (21.6\%) relapsed. In RPLND group, four of the 34 patients $(11.8 \%)$ relapsed. Positive lymph nodes were identified at surgery in 15 patients of the 34 patients (44.1\%) in RPLND group, and 12 patients received adjuvant chemotherapy after RPLND. Eighteen patients underwent adjuvant chemotherapy after primary orchiectomy, and only one member of this group relapsed (5.6\%). The cumulative 4 -year recurrence-free rates were $80.2 \%, 92.0 \%$ and $100 \%$ in the

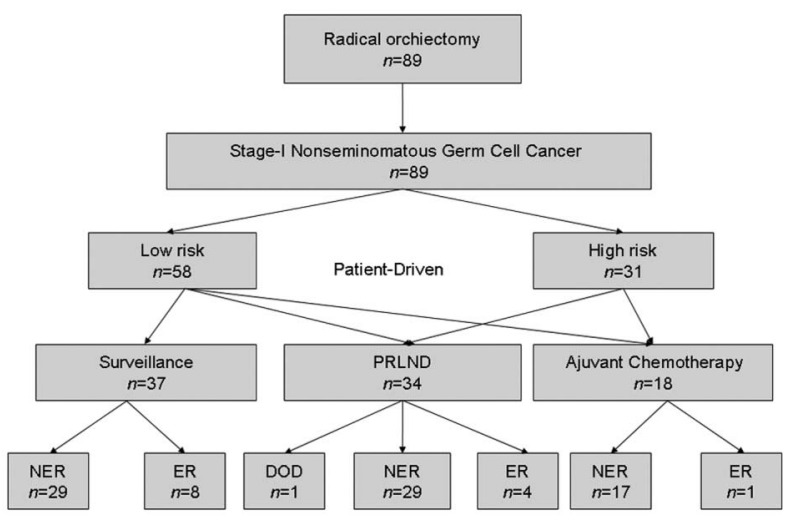

Figure 5 Recurrence-free survival for all patients of primary tumour stages la, Ib and Is.
Table 3 Multivariate analysis for different prognostic variable in patients with clinical stage I non-seminomatous germ cell tumours by Cox regression analysis

\begin{tabular}{lcc}
\hline Variable & OR $(95 \% \mathrm{Cl})$ & $\mathrm{P}$ \\
\hline Age at surgery & $(0.987,1.128)$ & 0.117 \\
Primary tumour clinic stage & $(1.008,8.522)$ & 0.048 \\
Tumour local side & $(0.593,6.820)$ & 0.262 \\
Initial treatment after orchiectomy & $(0.101,0.855)$ & 0.025 \\
History of cryptorchidism & $(0.043,0.581)$ & 0.005 \\
\hline
\end{tabular}

Abbreviations: $\mathrm{Cl}$, confidence interval; OR, odds ratio.

surveillance, RPLND and adjuvant chemotherapy groups, respectively $(P=0.441)$.

Figure 4 presents the recurrence-free survival rate for patients with a history of cryptorchidism treated with surveillance, RPLND and adjuvant chemotherapy. In the cryptorchidism group, four of the six patients $(66.7 \%)$ relapsed, and the cumulative 5-year recurrencefree rate was $61.3 \%(P=0.023)$.

The recurrence-free survival rates for the patients with different primary tumour stages treated using the three treatments are shown in Figure 5. The disease-free period was lower in the stage Is patients. The cumulative 4-year recurrence-free rate for primary tumour stages Ia and $\mathrm{Ib}$ were $100 \%$ and $84.7 \%$, respectively $(P<0.001)$.

In the multivariate analysis of the prognostic variables, the prognostic factors included a history of cryptorchidism (odd ratio $95 \%$ confidence intervals: $0.043-0.581, P=0.005)$ and initial treatment after orchiectomy (odd ratio 95\% confidence intervals: $0.101-0.855$, $P=0.025$; Table 3)

With regard to sexual function, we excluded those $(n=30)$ who preoperatively reported experiencing severe $\mathrm{ED}$, baseline International Index of Erectile Function 5-item score $(\leqslant 7)$ and those who lacked relevant information needed to assess postoperative erectile function. Of the 59 men evaluated, 12 patients (20.3\%) experienced ED 2 months after orchiectomy. The proportions of patients who were reported experiencing ED in the surveillance, RPLND and adjuvant chemotherapy were $5 / 23$ (21.7\%), 4/22 (18.2\%) and 3/14 $(21.4 \%)$, respectively. These numbers showed a decreasing trend, but it was not significant ( $P=0.102$, chi-square test).

\section{DISCUSSION}

Currently, surveillance, RPLND and adjuvant chemotherapy produce cure rates of above $90 \%$ for patients with early-stage NSGCT. ${ }^{18}$ Our study confirmed this figure by finding a 5 -year overall survival rate of 98.9\%. Several factors (e.g., histopathology, the disease stage, serum biomarkers, the risk of relapse, immediate and delayed toxicity, the treatment cost and the preferences of the doctor and patient) dictate how individual cases of testicular cancer are treated. In China, the selection of the most appropriate treatment varies across different hospitals. However, the European Association of Urology, the European Germ Cell Cancer Consensus Group and the American NCCN have provided guidelines with respect to the management of CSI NSGCT. In the United States, RPLND remains the standard treatment for most hospitals. In Europe, adjuvant chemotherapy for high-risk patients and surveillance for low-risk patients are more common. ${ }^{4,11,19}$

The advantage of the surveillance strategy is that up to $86 \%$ of patients do not need additional treatment, and relapses can be cured in approximately $100 \%$ of cases. In our study, eight patients relapsed, and the cumulative 4 -year recurrence-free rate was $80.2 \%$. Patient's compliance and psychological distress regarding the recurrence 
rate might be the problems. The surveillance strategy can only be performed when sufficient diagnostic skill and equipment are offered and when patients' compliance is guaranteed. ${ }^{20}$

RPLND remains the gold standard for the pathologic staging of the retroperitoneum and an effective therapy for patients with minimal nodal involvements. This treatment can remove chemoresistant teratomas because its biological potential is unpredictable. Under ideal conditions, RPLND provides excellent results (i.e., 90\% survival with low long-term toxicity). ${ }^{21}$ However, patients might be exposed to surgery-associated side effects such as retrograde ejaculation. The relapse rate after RPLND is between $5.8 \%$ and $21 \%$ in the literature. ${ }^{22}$ In our study, four patients (11.8\%) had relapsed and one patient had died of liver cancer by the evaluation time.

Chemotherapy is generally the preferred treatment for high-risk disease. Its major advantage is that the rate of tumour recurrence is reduced to less than or equal to $5 \% .^{23}$ The major disadvantage is the potential overtreatment of up to $70 \%$ of unselected stage I patients. The short-term side effects of chemotherapy include nausea and vomiting which can be managed with potent clinical agents; the long-term side effects are decreased fertility and secondary malignancies. ${ }^{24,25}$ Late toxicity and its preventive measures should be adequately addressed with patients. Previous studies have shown that secondary malignancies have emerged as the leading cause of death among testicular cancer survivors. ${ }^{26,27}$ In our study, only one of the 18 patients with chemotherapy relapsed (5.6\%). The cumulative 4 -year recurrence-free rate was $100 \%$, and grade $3 / 4$ chemotherapy-related toxicity was not found. These findings validate the current use of BEP as an effective treatment to avoid relapses. However, approximately $70 \%$ of patients undergo unnecessary treatments when chemotherapy is applied without adequately selecting patients at high risk for progression. ${ }^{28}$

The known prognostic factors of testicular cancer include the presence of embryonal carcinoma or undifferentiated cells and vascular or lymphatic invasion. Other predictors of recurrence include the tumour stage, the primary tumour histopathological criteria, the serum tumour marker levels, cell cycle features and immunohistochemical stains such as p53, bcl-2, MIB1 and ip12. ${ }^{29}$ Our multivariate analysis revealed that, having a history of cryptorchidism and a initial treatment after orchiectomy predicted relapse. The log-rank analysis also revealed a difference between the patients who had a history and those who had no history of cryptorchidism with regard to recurrence-free survival. Previous studies have reported that men with a history of cryptorchidism are 5-10 times more likely to contact testicular cancer than those who do not have this history. A history of cryptorchidism not only predicts relapse but also guides the optimal treatment and indicates prognosis.

Another finding is that the stage Is patients had a briefer disease-free period and the surveillance treatment had a higher recurrence rate than RPLND and chemotherapy. Stage Is NSGCT is an important indicator in therapy due to the high relapse rate. Davis et al. ${ }^{30}$ reported that 11 patients with stage Is NSGCT underwent primary RPLND at Memorial Sloan-Kettering Cancer Centre. All 11 patients subsequently and systemically relapsed during the follow-up period. Certain centers have recommended induction chemotherapy for clinical practice. Usually, three cycles of BEP are needed. The rising levels of $\alpha$-fetoprotein and beta-human chorionic gonadotropin must be interpreted with caution (except in cases of disseminated nonseminoma) because they might have other causes, such as hepatobiliary disease. $^{30}$

Because the disease affects relatively young men and the survival rate has increased, the awareness of long-term sequelae has advanced.
In a prospective study, ED occurred in $12.1 \%$ of NSGCT patients. ${ }^{11}$ In our study, more than $20 \%$ of the patients experienced ED after treatment. Treatment alternatives do not significantly influence ED. Both physiological changes and psychological factors cause sexual problems. A diagnosis of cancer is threatening and has long-lasting negative psychological effects on patients. Adequate information and support may prevent or reduce unnecessary sexual and relational anxieties and suffering.

Finally, the current trial is incomplete and awaits additional study. For instance, the indication for the chosen treatment strategies could not be determined from the retrospectively assessed data. The small number of patients might have affected the reliability and confidentiality of the results. Childhood ( $<14$ years of age) and adults with CSI NSGCT were included in this study; however, this sample is not appropriate because the histologies of these tumours tend to be different. Only ED was evaluated with regard to sexual function after treatment. More life quality problems should be considered in future studies.

In the light of our results, a strategy to identify genetic and molecular predictors will be useful to better select patients who will benefit from adjuvant therapy and avoid overtreatment.

\section{CONCLUSION}

This retrospective report is the first to study the clinical outcomes of the different management modalities among patients with CSI NSGCT from 1997 to 2011 in Guangdong, China. The results showed that surveillance, RPLND and adjuvant chemotherapy are all reliable treatment strategies. The disease-free period tended to be briefer in the stage Is patients and those with a history of cryptorchidism. Understanding the outcomes and long-term sequelae in survivors remains an important area of research.

Choosing the correct adjuvant approach in patients with CSI NSGCT must be related to individual prognostic factors and the care capacity of the treating centre. To provide the optimal treatment for every patient, the national standard therapy must be established and strictly followed. A comparison with reports from other parts of China might serve as a guide for the national management of the disease in the future.

\section{AUTHOR CONTRIBUTIONS}

ZJL, SW and ZWL designed the study and drafted the manuscript. PD, $\mathrm{KY}$ and YYH collected and analysed the data. YTG revised the manuscript and designed the experiments. ZMC, ZWL and FJZ conceived and designed the study. All the authors read and approved the final manuscript.

\section{COMPETING FINANCIAL INTERESTS}

All authors declare that there are no competing financial interests.

\section{ACKNOWLEDGMENTS}

Sun Yat-Sen University Cancer Center, Shenzhen PKU-HKUST Medical Center and the First Affiliated Hospital of Shenzhen University supported this study. This work was supported by grants from the Chinese High Tech (863) Program (Nos. 2012AA02A201 and 2012AA02A208) and the National Natural Science Foundation of China (No. 81071704).

1 Huyghe E, Matsuda T, Thonneau P. Increasing incidence of testicular cancer worldwide: a review. J Urol 2003; 170: 5-11.

2 Siegel R, Naishadham D, Jemal A. Cancer statistics, 2012. CA Cancer J Clin 2012; 62: $10-29$ 
3 Garner MJ, Turner MC, Ghadirian P, Krewski D. Epidemiology of testicular cancer: an overview. Int J Cancer 2005; 116: 331-9.

4 National Comprehensive Network. NCCN Clinical Practice Guidelines in Oncology Testicular Cancer, version 1. Fort Washington, PA: NCCN; 2012.

5 Brydoy M, Fossa SD, Klepp 0, Bremnes RM, Wist EA et al. Paternity following treatment for testicular cancer. J Natl Cancer Inst 2005; 97: 1580-8.

6 de Wit R, Fizazi K. Controversies in the management of clinical stage I testis cancer. J Clin Oncol 2006; 24: 5482-92.

7 Tandstad T, Dahl O, Cohn-Cedermark G, EvaCavallin-S, Ulrika S et al. Risk-adapted treatment in clinical stage I nonseminomatous germ cell testicular cancer: the SWENOTECA management program. J Clin Oncol 2009; 27: 2122-8.

8 Williams SB, McDermott DW, Winston D, Bahnson EM, Berry A et al. Retroperitoneal lymph node dissection in patients with high risk testicular cancer. J Urol 2009; 181: 2097-102.

9 Stephenson AJ, Bosl GJ, Motzer RJ, Bajorin DF, Stasi JP et al. Nonrandomized comparison of primary chemotherapy and retroperitoneal lymph node dissection for clinical stage IIA and IIB nonseminomatous germ cell testicular cancer. J Clin Oncol 2007; 25: 5597-602.

10 Beck S, Peterson MD, Bihrle R, Donohue JP, Foster RS et al. Short term morbidity of primary retroperitoneal lymph node dissection in a contemporary group of patients. J Urol 2007; 178: 504-6.

11 Krege S, Beyer J, Souchon R, Albers P, Albrecht W et al. European consensus conference on diagnosis and treatment of germ cell cancer: a report of the second meeting of the European Germ Cell Cancer Consensus group (EGCCCG): Part I. Eur Urol 2008; 53: 478-96.

12 Tarin TV, Sonn G, Shinghal R. Estimating the risk of cancer associated with imaging related radiation during surveillance for stage I testicular cancer using computerized tomography. J Urol 2009; 181: 627-32.

13 Howard R, Gilbert E, Lynch CF, Hall P, Storm H et al. Risk of leukemia among survivors of testicular cancer: a population-based study of 42,722 Patients. AEP 2008; 18 : 416-21.

14 Haugnes HS, Aass N, Fossa SD, Dahl O, Brydøy M et al. Pulmonary function in longterm survivors of testicular cancer. J Clin Oncol 2009; 27: 2779-86.

15 Jonker-Pool G, van de Wiel HB, Hoekstra HJ, Sleijfer DT, van Driel MF et al. Sexual functioning after treatment for testicular cancer-review and meta-analysis of 36 empirical studies between 1975-2000. Arch Sex Behav 2001; 30: 55-74.

16 Bhayani SB, Ong A, Oh WK, Kantoff PW, Kavoussi LR et al. Laparoscopic retroperitoneal lymph node dissection for clinical stage I nonseminomatous germ cell testicular cancer: a long-term update. Urology 2003; 62: 324-7.
17 Albers $\mathrm{P}$, Siener R, Kliesch S, Weissbach L, Krege S et al. Risk factors for relapse in clinical stage I nonseminomatous testicular germ cell tumors: results of the German Testicular Cancer Study Group Trial. J Clin Oncol 2003; 15: 1505-12.

18 Steele GS, Richie JP, Stewart AK, Menck HR. The National Cancer Data Base report on patterns of care for testicular carcinoma, 1985-1996. Cancer 1999; 86: 2171-83.

19 European Association of Urology. Guidelines on Testicular Cancer. Arnhem: European Association of Urology; 2012.

20 van As NJ, Gilbert DC, Money-Kyrle J, Bloomfield D, Beesley S et al. Evidence-based pragmatic guidelines for the follow-up of testicular cancer: optimising the detection of relapse. Br J Cancer 2008; 98: 1894-902.

21 Poulakis V, Skriapas K, de Vries R, Dillenburg W, Ferakis N et al. Quality of life after laparoscopic and open retroperitoneal lymph node dissection in clinical Stage nonseminomatous germ cell tumor: a comparison study. Urology 2006; 68: 154-60.

22 Stephenson AJ, Bosl GJ, Bajorin DF, Kattan MW, Stasi J et al. Retroperitoneal lymph node dissection in patients with low stage testicular cancer with embryonal carcinoma predominance and/or lymphovascular invasion. J Urol 2005; 174: 557-60.

23 Bokemeyer C, Schmoll HJ, Kuczyk MA, Einhorn LH. Risk of secondary leukemia following high cumulative doses of etoposideduring chemotherapy for testicular cancer. J Natl Cancer Inst 1995; 87: 58-60.

24 Chevreau C, Mazerolles C, Soulié M, Gaspard MH, Mourey L et al. Long-term efficacy of two cycles of BEP regimen in high-risk stage I nonseminomatous testicular germ cel tumors with embryonal carcinoma and/or vascular invasion. Eur Urol 2004; 46: 209-14.

25 Pont J, Albrecht W. Fertility after chemotherapy for testicular germ cell cancer. Ferti Steril 1997; 68: 1-5.

26 Fossa SD, Aass N, Harvei S, Tretli S. Increased mortality rates in young and middleaged patients with malignant germ cell tumours. Br J Cancer 2004; 90: 607-12.

27 Zagars GK, Ballo MT, Lee AK, Strom SS. Mortality after cure of testicular seminoma. J Clin Oncol 2004; 22: 640-7.

28 Tandstad T, Dahl O, Cohn-Cedermark G, Cavallin-Stahl E, Stierner U et al . Risk adapted treatment in clinical stage I nonseminomatous germ cell testicular cancer: the SWENOTECA Management Program. J Clin Oncol 2009; 27: 2122-8.

29 Albers P, Orazi A, Ulbright TM, Miller GA, Haidar JH et al. Prognostic significance of immunohistochemica proliferation markers (Ki-67/MIB-1 and proliferation-associated nuclear antigen) p53 protein accumulation, and neovascularization in clinical stage $A$ nonseminomatous testicular germ cell tumors. Mod Pathol 1995; 8: 492-7.

30 Davis BE, Herr HW, Fair WR, BosI GJ. The management of patients with nonseminomatous germ cell tumors of the testis with serologic disease only after orchiectomy. J Urol 1994; 152: 111-3. 